\title{
Consideraciones sobre la Espiritualidad y la Religión como Recursos de Afrontamiento durante la Pandemia del COVID-19
}

\section{Considerations on Spirituality and Religion as Coping Resources during the COVID-19 Pandemic}

Eunice Alvarado-Díaz 1* ${ }^{*}$ \& Orlando M. Pagán-Torres ${ }^{2}$

1 Ponce Health Sciences University, Ponce, Puerto Rico. (D) https://orcid.org/0000-0002-0575-2194

2 Carlos Albizu University, San Juan, Puerto Rico. (D) https://orcid.org/0000-0002-7195-4202

* Correspondencia: 388 Zona Industrial Reparada 2, Ponce, PR 00716. Email: ealvarado@psm.edu.

Recibido: 15 agosto 2020 | Aceptado: 31 marzo 2021 | Publicado: 3 abril 2021

WWW.REVISTACARIBENADEPSICOLOGIA.COM

\section{Citar como:}

Alvarado-Díaz, E., \& Pagán-Torres, O. M. (2021). Consideraciones sobre la espiritualidad y la religión como recursos de afrontamiento durante la pandemia del COVID-19. Revista Caribeña de Psicología, 5, e5007. https://doi.org/10.37226/rcp.v5i1.5007

\section{RESUMEN}

La pandemia del COVID-19 se ha convertido en objeto de atención que ha amenazado la salud pública en múltiples formas, por lo que inevitablemente ha trastocado la comunidad religiosa. La asociación entre espiritualidad, religión (E/R) y salud ha sido empíricamente documentada en la literatura científica de salud mental. Dada la importancia de la E/R en la salud integral de las personas, el objetivo de este artículo consiste en exponer una serie de consideraciones relevantes sobre la E/R en el afrontamiento positivo y negativo de la pandemia del COVID-19 y el distanciamiento social. Además, se discuten los hallazgos recientes sobre la E/R y COVID-19, factores relevantes para la integración de la E/R en la psicoterapia y diversas técnicas psicoterapéuticas de base religiosa y espiritual que han mostrado ser eficaces para el manejo adaptativo de eventos estresantes y situaciones de crisis. Esta discusión plantea la importancia de desarrollar competencias en el campo de la psicología de la religión y la espiritualidad con el objetivo de conceptualizar cómo estas dimensiones podrían impactar el bienestar psicológico de las personas en situaciones de angustia y crisis. Finalmente, se discute la importancia de considerar la dimensión espiritual y religiosa como recursos de afrontamiento en situaciones de crisis e incertidumbre.

Palabras Claves: afrontamiento; COVID-19; espiritualidad; pandemia; religión

\begin{abstract}
The COVID-19 pandemic has become an object of attention that has threatened public health in multiple ways, inevitably disrupting the religious community. The association between spirituality, religion (S/R), and health has been empirically documented in the scientific literature on mental health. Given the importance of S/R in people's health, this article's objective is to expose a series of relevant aspects about $S / R$ in positive and negative coping of the COVID-19 pandemic and social distancing. Also, it is discussed recent findings on S/R and COVID-19, relevant
\end{abstract}


factors for the integration of S/R in psychotherapy, and various psychotherapeutic techniques with a religious and spiritual base that are effective for adaptive management of stressful and crises. This discussion exposes the importance of developing competencies in the field of psychology of religion and spirituality to conceptualize how these dimensions could impact people's psychological well-being in distress and crises. Finally, we discuss the importance of considering the spiritual and religious dimensions as coping resources in crisis and uncertain situations.

Keywords: coping; COVID-19; pandemic; religion; spirituality

\section{INTRODUCCIÓN}

La enfermedad del coronavirus surgida en el 2019 (COVID-19) se ha convertido en objeto de atención que ha amenazado la salud pública en todo el mundo. Se ha sugerido que la incidencia de desórdenes mentales durante la pandemia del COVID-19 no es únicamente explicada por el miedo al contagio del virus, sino también al distanciamiento social (Hossain et al., 2020a). Entre las condiciones de salud mental más prevalentes que se han identificado debido a la crisis del COVID-19 se encuentran la depresión, ansiedad, estrés postraumático (TEPT), angustia psicológica y pensamientos/intentos suicidas (Hoassin et al., 2020b). El incremento de estas condiciones durante la actual pandemia concurre con diversos estudios que sugieren que la incidencia de trastornos mentales, después de un mes de ocurrido el desastre o evento natural, es significativa (Beaglehole et al., 2018). Esto hace relevante el estudio científico de las estrategias de afrontamiento para el manejo adaptativo de pensamientos y conductas maladaptativas causados por el miedo a la pandemia y el distanciamiento social (Loreno-Ruiz et al., 2020).

Por otra parte, la asociación entre religión, espiritualidad (E/R) y salud ha sido sistemáticamente documentada en la literatura científica de salud mental (Bonelli et al., 2013; Koenig, 2009; 2015, 2020; Paloutzian et al., 2013). Alrededor de 3,300 investigaciones cuantitativas publicadas desde el siglo 19 hasta el 2010 revelan la importancia de la dimensión religiosa y espiritual para la salud en personas con creencias religiosas y espirituales (Koenig, 2012). Recientemente, una revisión de más de 100 metaanálisis y revisiones sistemáticas de literatura documenta el impacto positivo del involucramiento religioso en la salud mental y salud pública (Oman \& Syme, 2018). No obstante, cabe resaltar que este hallazgo es más robusto en países donde la gente es eminentemente religiosa (e.g., Estados Unidos y países de Latinoamérica, África y Asia). En países más seculares, como algunos de Europa, estas diferencias se minimizan o desaparecen (Lun \& Bond, 2013).

A pesar de las limitaciones metodológicas documentadas en los estudios de E/R (de-Oliveira-Maraldi, 2020; Koenig, 2008; 2018), la asociación positiva entre dichas dimensiones en la salud mental ha sido robusta en la literatura empírica (Koenig, 2018; Park, 2017). Por consiguiente, la $E / R$ pueden desempeñar roles significativos com1o variables protectoras de la salud mental al proveer estrategias adaptativas para el manejo de situaciones de crisis e incertidumbre (Pargament et al., 2013). Dada la importancia de la $\mathrm{E} / \mathrm{R}$ en la salud mental y física, el objetivo de este artículo consiste en exponer una serie de consideraciones relevantes de la $\mathrm{E} / \mathrm{R}$ en el afrontamiento de la pandemia del COVID-19 y el distanciamiento social. Para cumplir este objetivo, se discutirán los hallazgos recientes sobre la $\mathrm{E} / \mathrm{R}$, síntomas psicológicos debido a la pandemia, factores relevantes para la integración de la E/R en la psicoterapia, así como discusión de algunas técnicas psicoterapéuticas de base religiosa y espiritual que podrían ser de utilidad para el manejo adaptativo de la crisis del COVID-19.

\section{Espiritualidad, Religión y Trauma}

Horesh y Brown (2020) exponen cómo el COVID19 ha impactado las infraestructuras físicas y emociones del mundo entero, aumentando la vulnerabilidad de las personas y generando una crisis a nivel global. Van-Hook (2016) destaca que una "potencial amenaza" puede generar diversas emociones y pensamientos, produciendo temor e indefensión que pueden conducir a trauma. Precisamente, Horesh y Brown (2020) señalaron que la crisis del COVID-19 debía ser abordada desde el punto de vista del trauma. El concepto de trauma y la guía para un enfoque basado en el trauma de la Administración de 
Servicios de Salud Mental y Abuso de Sustancias (SAMHSA, por sus siglas en inglés) definen el trauma individual como "el resultado de un evento, una serie de eventos o un conjunto de circunstancias que un individuo experimenta como física o emocionalmente dañino o potencialmente mortal y que tiene efectos adversos duraderos en el funcionamiento y el funcionamiento mental, físico, social, emocional o bienestar espiritual del individuo" (SAMHSA, 2014, p.7).

De acuerdo con esta definición, y tomando en consideración que el COVID-19 es una pandemia, son muchas las personas que cumplen con las características para considerar este evento como dañino o potencialmente mortal (Griffin, 2020). Sin embargo, es importante resaltar que no todas las personas poseen estrategias de afrontamiento adaptativas para el manejo saludable de los factores estresantes producidos por la pandemia del COVID-19 (SAMSHA, 2014). Esto sugiere que podría haber individuos que no necesariamente desarrollarán síntomas de trauma o necesitarán servicios de salud mental, mientras que otros podrían experimentar un mayor impacto psicológico, desarrollando síntomas asociados a experiencias traumáticas (Griffin, 2020; SAMSHA, 2014). Por su parte, la revisión de Van-Hook (2016) expone que las experiencias traumáticas pueden impactar el cuerpo, pensamientos, emociones, así como la vida interpersonal y espiritual de las personas. Estos efectos llegan a convertirse en una responsabilidad no solo para las personas que experimentaron las experiencias traumáticas, sino también para sus familias (Horesh \& Brown, 2020). Más aún, cuando una persona con síntomas postraumáticos no recibe los servicios de salud mental necesarios, podría aumentar el riesgo de trastornos mentales comórbidos como el uso de sustancias, depresión y enfermedades físicas crónicas (SAMHSA, 2014).

Todo esto refleja la importancia de abordar el trauma desde un enfoque multidisciplinario (SAMSHA, 2014) que integre el aspecto espiritual y religioso (Richard \& Bergin, 2005; 2014). Kick y McNitt (2016) catalogaron como "buena práctica" explorar el aspecto espiritual, así como el sistema de creencias de la persona que recibirá servicios de salud mental. Por ejemplo, la espiritualidad, la fe y la religión pueden ser percibidas como fuentes de apoyo en veteranos y sus familiares (Kick et al., 2016). Ciertamente, diversos estudios reconocen la $\mathrm{E} / \mathrm{R}$ como recurso $\mathrm{y}$ estrategia de afrontamiento para el manejo de situaciones de angustia o de trauma (Starnino, 2016; Koenig; 2012). Además, las dimensiones espiritual y religiosa son vistas como recursos en el aspecto social, al proveer redes de apoyo en situaciones cotidianas (Currier \& Eriksson, 2017; Pearce et al., 2015a; 2016). De manera similar, Van-Hook (2016) explica que la espiritualidad puede ayudar a las personas a recuperarse luego de alguna situación difícil al generar alivio, esperanza y sentido de valor. Además, añade que la espiritualidad está relacionada con el sentido de significado. Esto puede llevar a las personas a que encuentren en la E/R la oportunidad de recuperar su significado de vida, retomando el control de esas situaciones llegando a observarlas como eventos con propósito (Stephens et al., 2013).

La literatura presenta diversas prácticas espirituales y religiosas que resultan ser de apoyo para personas con experiencias de trauma. Son varios los autores que resaltan que prácticas como la oración, lectura de textos sagrados y el apoyo de una congregación pueden aliviar los síntomas de angustia del trauma, generando bienestar en las personas con estas experiencias (Currier et al., 2017; Park et al., 2017; Richard \& Bergin, 2005; 2014). Precisamente, Kick et al. (2016) destacan que la oración junto a la meditación ha sido utilizada como estrategia de afrontamiento para los síntomas de TEPT, en militares. Con relación a esto, Currier et al. (2017) presentan cómo las normas de conductas que existen en las religiones tradicionales fomentan un estilo de vida moral y saludable. Según estos autores, la $\mathrm{E} / \mathrm{R}$ pueden ser recursos de apoyo para disminuir conductas maladaptativas que podrían estar presentes en las personas con experiencias de trauma y abuso de alcohol y drogas.

Por otro lado, es importante destacar que las prácticas espirituales y religiosas pueden asociarse con un impacto negativo en la salud mental a través del afrontamiento religioso negativo y la presencia de luchas religiosas y espirituales (Ano \& Vasconcelles, 2005; Ano et al., 2013). El trauma o la situación adversa pueden impactar la espiritualidad de los individuos (Van-Hook, 2016; Van-Tongeren et al., 2019) asociándose con cuestionamientos a la existencia de una divinidad o ser supremo (Bradley et al., 2018; Exline, 2013). También, puede estar relacionado con disminución en la fe, ateísmo (Aten et. al, 2019, Currier \& Eriksson, 2017; Sedlar et al., 2018) y mayor angustia 
psicológica (Abu-Raiya et al., 2015). Ciertamente, no se debe ignorar que las experiencias de trauma a nivel individual o de comunidad (como el COVID-19), pueden tener un impacto en el significado de vida de las personas (Aten et al., 2019). Incluso, se han identificado sobrevivientes que tras experiencias de trauma reformulan su propósito de vida (Peres et al., 2007).

Un ejemplo de lucha religiosa/espiritual sería el de un individuo que ha sido profundamente religioso y espiritual durante muchos años y de momento experimente diversas pérdidas familiares significativas debido al COVID-19. A partir de esto, la persona comienza a tener cuestionamientos religiosos que ponen en duda la fidelidad, el amor e incluso la existencia de la divinidad debido a la maldad y el sufrimiento en el mundo. Incluso, algunas personas pueden llegar a experimentar un sentido de soledad espiritual, denominado por algunos como el "ocultamiento de Dios". Estas personas pueden experimentar lo que ha sido reconocido como afrontamiento religioso negativo. Personas que utilizan este tipo de afrontamiento suelen sentirse abandonados por la divinidad y la comunidad religiosa; se sienten perseguidos o atacados por espíritus demoniacos, dudan del amor divino, etcétera (Pargament, 1997). Tales pensamientos, en una persona que haya sido profundamente religiosa durante el transcurso de su vida podrían provocar un conflicto en sus eventos internos y privados debido a cuestiones religiosas. Estos cambios en sus percepciones, forma de ver la vida y el proceso de desconversión podrían ser un factor de riesgo que aumente la vulnerabilidad de experimentar síntomas psicológicos en algunas personas religiosas y espirituales.

\section{Espiritualidad, Religión y COVID-19}

La pandemia del COVID-19 ha trastocado la comunidad religiosa en múltiples formas, siendo una de estas la organización y métodos para continuar realizando los servicios religiosos. Incluso, se ha llegado a sugerir la importancia de regular los servicios religiosos con el objetivo de prevenir la propagación de la pandemia (Quadri, 2020). Sulkowski et al. (2020) entrevistó 12 clérigos (sacerdotes y pastores) para evaluar qué tipo de actividades son realizadas en las iglesias cristianas en Polonia durante la pandemia de COVID-19 y explorar las diferencias en su organización religiosa. Los investigadores encontraron que las iglesias tienen limitado (católicos y ortodoxos) o totalmente suspendido (protestantes) su vida religiosa en comunidad. A pesar del cambio en la organización religiosa, las iglesias mantuvieron contacto con los creyentes a través de recursos tecnológicos y acceso a los medios públicos. También, los resultados revelaron que las iglesias no han cambiado sus posiciones doctrinales debido a la crisis del COVID-19.

Por otra parte, Fardin (2020) revisó los artículos publicados desde 1978 al 2019 sobre la relación entre la espiritualidad en tiempos de crisis. Los resultados revelaron que la espiritualidad puede ayudar a las personas a tener relajación mental en tiempos de crisis y enfermedades peligrosas. También, Pirustinsky et al. (2020) evaluaron el impacto de la pandemia y exploraron las relaciones entre exposición, religiosidad y angustia en una muestra de 419 judíos ortodoxos estadounidenses. Los resultados indicaron altos niveles de exposición, preocupación y cumplimiento con pautas médicas. Sin embargo, los hallazgos sugieren un impacto positivo del afrontamiento religioso positivo, religiosidad intrínseca y confianza en Dios con menos estrés, mientras que el afrontamiento religioso negativo y desconfianza en Dios correlacionaron positivamente con estrés.

Por otra parte, la Organización Mundial de la Salud (2020) ha expuesto una serie de recomendaciones dirigidas a líderes religiosos y a comunidades de base de fe para promover la prevención del contagio del COVID-19 en los templos tales como: (1) mantener al menos tres pies de distancia entre las personas en todo momento, (2) evitar tocarse entre personas que asisten a servicios religiosos, evitar tocar o besar devocionales y otros objetos que la comunidad está acostumbrada a manejar comunalmente, (3) fomentar una higiene saludable entre los participantes de los servicios religiosos y otras actividades, (4) limpiar con frecuencia los espacios de servicios religiosos y (5) realizar actividades de fe de forma remota/virtual.

Además, Koenig (2020) recientemente expuso una variedad de conductas adaptativas con enfoque espiritual que las personas religiosas pueden realizar para disminuir el riesgo del contagio de la pandemia y para manejar adaptativamente los efectos del virus en una persona contagiada por el mismo. Entre las recomendaciones se encuentran las siguientes: (1) profundizar en la fe religiosa, (2) amar al prójimo como a sí 
mismo, (3) usar la tecnología, (4) amar y cuidar al prójimo en formas prácticas, (5) no ser temerario y (6) prestar atención a la salud física.

\section{FACTORES RELEVANTES SOBRE LA INTE- GRACIÓN DE LA ESPIRITUALIDAD Y RE- LIGIÓN EN LA PSICOTERAPIA}

Existen diversos factores a considerar en la integración de la E/R en el escenario psicoterapéutico: (1) eficacia, (2) actitudes de los pacientes/clientes, (3) actitudes y competencias de los profesionales de la salud mental y (4) asuntos éticos. Consideramos estos elementos fundamentales para el éxito de la integración de la $\mathrm{E} / \mathrm{R}$ y el bienestar del paciente/cliente durante el tratamiento psicológico.

Con relación a la eficacia de las técnicas psicoterapéuticas de base espiritual y religiosa, vale la pena enfatizar que un conjunto de revisiones de literatura y metaanálisis han sostenido la importancia de su integración en psicoterapia (Anderson et al., 2015; Gonçalves et al., 2015; Hook et al., 2010; Lim et al., 2014; Oh \& Kim, 2012; Paukert et al., 2011; Tan et al., 2005). Por ejemplo, el metaanálisis de Smith et al., (2007) sugiere que las terapias orientadas espiritualmente pueden ser eficaces para personas con síntomas psicológicos asociados a depresión, ansiedad y desórdenes alimenticios. También, Gonçalves et al. (2015) realizaron una revisión sistemática y metaanálisis de ensayos controlados aleatorizados que documentan que las intervenciones psicoterapéuticas religiosas y espirituales han mostrado una reducción significativa en síntomas de ansiedad. Sin embargo, en este metaanálisis solo el $26 \%$ de los estudios estaban asociados a salud mental. Estos hallazgos son relevantes para el desarrollo e implementación de técnicas psicoterapéuticas de base espiritual y religiosa en el escenario psicoterapéutico.

El metaanálisis de Worthington y colaboradores (2011) compararon las intervenciones que incluían E/R contra una psicoterapia secular controlando por orientación teórica y duración. Los resultados no encontraron una diferencia notable entre los dos tratamientos $(d=.13)$. Recientemente, Captari et al., (2018) realizaron un metaanálisis donde incluyeron 7,181 participantes con el objetivo de examinar la eficacia de adaptar al tratamiento las creencias y valores de $\mathrm{E} / \mathrm{R}$ de los pacientes. Se comparó la efectividad de la psicoterapia adaptada a $E / R$ con controles sin tratamiento, tratamientos seculares alternativos y tratamientos seculares aditivos. La psicoterapia adaptada a E/R resultó en una mayor mejoría en el funcionamiento psicológico $(g=0,74, p<0,000)$ y espiritual $(g=0,74, p<0,000)$ de los clientes en comparación con las psicoterapias sin tratamiento y sin $\mathrm{E} / \mathrm{R}$ (psicológico: $g=0,33, p<0,001$; espiritual: $g=0,43, p<0,001$ ). En estudios aditivos más rigurosos, las psicoterapias adaptadas a E/R fueron igualmente efectivas que los enfoques estándar para reducir la angustia psicológica $(g=0,13, p=0,258)$. Esto sugiere importantes coincidencias en estudios que indican que los que más se benefician de intervenciones en donde se incorpora la E/R son las personas con puntuaciones altas en religiosidad.

Recientemente, varios investigadores/as en los Estados Unidos han desarrollado un modelo de tratamiento conocido como Terapia Cognitivo Conductual Religiosamente Integrada (TCCRI) (Pearce et al., 2015a). Los investigadores realizaron un ensayo clínico aleatorizado con 132 pacientes de 18 a 85 años diagnosticados con depresión mayor y condiciones médicas. Los participantes fueron asignados aleatoriamente a uno de dos tratamientos: la TCCRI o la Terapia Cognitivo Conductual Estándar (TCCE). Los estudios revelaron que, en la muestra general, la TCCRI fue tan efectiva como TCCE en generar sentido de gratitud (Pearce et al., 2015b), optimismo (Koenig et al., 2015) y propósito de vida (Daher et al., 2016). Además, resultaron igualmente efectivas en disminuir pensamientos suicidas (Ramos et al., 2018). Estos hallazgos sugieren que no existen diferencias significativas entre la TCCRI y la TCCE.

Por lo tanto, ¿cuáles son los beneficios de añadir elementos religiosos y espirituales a las terapias estándar? Primero, contar con estas psicoterapias integradas como alternativas podría resultar en una relación más sólida entre cliente y terapeuta, así como en mayor posibilidad de cambio (Wade et al., 2007). También, la honestidad de pacientes y la confianza de poder abrirse sin temor a ser juzgados puede influir en cuánto progreso obtienen de la terapia. Por eso, cuando las personas que valoran lo E/R participan de terapias que toman estas dimensiones en cuenta, podrían sentirse más seguros y confiados en abrirse y discutir en terapia los verdaderos asuntos que las afectan. Algunos clientes podrían considerar que hay una dimensión espiritual en sus problemas de vida, o 
piensen que estos afectan su espiritualidad (PagánTorres et al., 2017).

Con relación a las actitudes de los pacientes/clientes hacia la integración de la $\mathrm{E} / \mathrm{R}$ durante el tratamiento, se ha identificado que la mayoría de los estudios sugieren que los pacientes/clientes apoyan la discusión de la E/R durante el tratamiento de salud mental, particularmente entre aquellos quienes se consideran religiosos o espirituales (Harris et al., 2016). Oxhandler et al. (2018) exploraron las actitudes hacia la integración de la religión y la espiritualidad en el tratamiento de salud mental en 1,047 adultos norteamericanos. Los resultados revelaron que la mayoría de los participantes mostraron una actitud favorable hacia la integración de la espiritualidad y religión en la psicoterapia. Recientemente, Yamada et al. (2020) evaluaron las actitudes hacia la inclusión de la espiritualidad en el tratamiento en 2,050 personas que reciben servicios de salud mental. Los investigadores identificaron que más del $80 \%$ de los participantes estuvieron de acuerdo en que la espiritualidad era un aspecto importante para su salud mental y mostraron apertura hacia su integración en el tratamiento. También, se ha reportado que del $77 \%$ al $83 \%$ de los pacientes mayores de 55 años desean tener sus creencias religiosas integradas en la terapia (Stanley et al., 2011).

Sobre las actitudes de los profesionales de la salud mental hacia la integración de la $\mathrm{E} / \mathrm{R}$ en la psicoterapia, la revisión de Post et al. (2009) sugiere que los psicoterapeutas suelen mostrar apertura a dialogar sobre asuntos religiosos y espirituales con sus pacientes/clientes. En Puerto Rico, alrededor del 96\% de los profesionales de la salud mental mostraron una actitud favorable hacia integración de la espiritualidad (González-Rivera, 2016). No obstante, se ha reportado que los psicólogos(as) suelen dialogar sobre $\mathrm{E} / \mathrm{R}$ con alrededor del $30 \%$ de sus pacientes/clientes durante el tratamiento psicológico (Hathaway et al., 2004; Saunders et al., 2010). Incluso, Best et al. (2016) identificaron en su revisión sistemática la falta de entrenamiento como una de las principales barreras entre los médicos para dialogar sobre asuntos de $\mathrm{E} / \mathrm{R}$ con sus pacientes. Por lo tanto, no es sorpresa que de forma similar también pueda ocurrir entre los profesionales de la salud mental.
Con relación a los asuntos éticos sobre la integración de la E/R en la psicoterapia, es importante destacar que existe apertura entre las principales asociaciones de psicología y consejería sobre la integración de la $\mathrm{E} / \mathrm{R}$ en la psicoterapia. Por ejemplo, la Asociación Americana de Psicología cuenta con la Sociedad de Psicología de la Religión y la Espiritualidad (División 36). Además, la Asociación Americana de Consejería cuenta con la Asociación de Valores Espirituales, Éticos y Religiosos en la Consejería (ASERVIC, 2009) la cual promueve la incorporación de la espiritualidad en la práctica de la consejería. Esto es importante, dado que los códigos de ética de la profesión de la psicología no prohíben la utilización de técnicas religiosas y espirituales en la psicoterapia siempre y cuando se haga responsablemente con fines psicoterapéuticos (González-Rivera, 2015; Pagán et al., 2017). También, la guía de consulta del Manual Diagnóstico y Estadístico de Trastornos Mentales-Quinta Edición (DSM-5) (American Psychiatric Association, 2013) contiene una categoría para ubicar problemas espirituales y religiosos que requieren de atención clínica conocido como: V62.89 Problema religioso o espiritual. Por tal razón, con el objetivo de poder atender la E/R en psicoterapia de forma competente y ética, Vieten et al. (2013) desarrollaron 16 competencias espirituales y religiosas que todo psicólogo(a) debería tener en el dominio de prácticas y creencias religiosas y espirituales. Dichas competencias están basadas en actitudes, conocimientos y destrezas que todo psicólogo(a) debe tener sobre la $E / R$.

\section{ESTRATEGIAS PSICOTERAPÉUTICAS DE BASE ESPIRITUAL Y RELIGIOSA}

El avance del estudio científico de la E/R en la salud mental ha innovado el desarrollo de técnicas psicoterapéuticas de base espiritual y religiosa que han mostrado ser beneficiosas como recursos de afrontamiento para el manejo adaptativo de eventos estresantes (Pearce, 2016; Rosmarin, 2018). Discutiremos algunas de las más reconocidas.

\section{La Oración}

La oración involucra dirección o comunicación con lo divino, siendo actualmente la práctica religiosa y espiritual más utilizada (Spilka et al., 2012). Existen cinco tipos de oraciones tales como: oración de agradecimiento, oración conversacional, oración de alabanza, oración de petición y oración de 
contemplación/silencio (Rosmarin, 2018). Investigaciones recientes sugieren que la oración se relaciona inversamente con angustia en personas que perciben a la divinidad como un ser cercano y amoroso (Ellison et al., 2014; Rosmarin et al., 2010). Prado et al. (2016) revisaron sistemáticamente investigaciones sobre el impacto de la oración en la salud. Un total de 92 artículos fueron identificados y 12 fueron incluidos en su revisión de literatura según sus criterios de inclusión. En siete de los doce estudios seleccionados, los investigadores identificaron varios efectos positivos de la oración sobre la salud tales como: la reducción de la ansiedad de las madres de niños con cáncer y reducción en el nivel de preocupación de los participantes que creen en una solución a su problema. Por lo tanto, es evidente que la oración y las creencias religiosas juegan un papel importante en el manejo adaptativo de eventos estresantes en las personas religiosas y espirituales.

Tan (1996) propone el uso de la oración de sanación interior en la sesión terapéutica. Resumimos su uso en los siguientes puntos: (1) el terapeuta comienza la oración pidiendo a la divinidad sanación interior, (2) el terapeuta debe ayudar al cliente a relajarse, (3) se ayuda al cliente a visualizar el trauma o herida, (4) se ora nuevamente a la divinidad, (5) se espera en silencio una respuesta de la divinidad (no literal ni audible); esto permite y facilita el procesamiento de la experiencia y sanación en el cliente, (6) se realiza una oración de cierre y (7) se discute con el cliente la experiencia vivida durante la oración. También, Koenig y Pritchett (1998) crearon un protocolo para el uso de la oración en el contexto psicoterapéutico. Resumimos el protocolo en los siguientes puntos: (1) El cliente debe verbalizar claramente que está de acuerdo con la técnica de la oración, (2) es preferible que el cliente sea quien haga la oración y el terapeuta escuche atentamente, (3) si el cliente pide al terapeuta que ore, la oración debe ser general, de apoyo, afirmativa y esperanzadora y (4) el terapeuta debe explorar la reacción del cliente a la oración y discutir las experiencias positivas y negativas.

Cabe resaltar que el uso de la oración con fines psicoterapéuticos debe ser específicamente dirigida a personas que posean creencias religiosas y espirituales congruentes con dicha práctica religiosa. Esto sugiere que la oración no se debe utilizar con personas en las que la $\mathrm{E} / \mathrm{R}$ no representen aspectos importantes en sus vidas. No es recomendable utilizar técnicas religiosas y espirituales con personas no-creyentes o con personas que no sean espirituales ni religiosas ya que podría afectar la alianza terapéutica y la satisfacción del cliente con los servicios. Antes de utilizar técnicas religiosas y espirituales con fines terapéuticos, se recomienda administrar al cliente la escala breve de afrontamiento religioso (Pargament et al., 1998).

\section{Ejercicios de Gratitud}

La gratitud es una afirmación de bondad en la cual una persona reconoce fuentes de beneficio fuera de sí mismo (Emmons et al., 2004). Jans-Beken et al. (2019) llevaron a cabo una revisión sistemática de todos los artículos publicados desde el 2010 hasta el 2018 sobre el impacto de la gratitud en la salud física y mental. Los resultados sugieren que la gratitud está asociada positivamente con mayor bienestar social, emocional y psicológico. No obstante, los efectos de la gratitud en la salud física no son consistentes. Por lo tanto, Boggis et al. (2020) realizaron una revisión sistemática sobre los efectos de las intervenciones de gratitud en la salud física y conductual. Los investigadores identificaron un total de 19 estudios de intervenciones basadas en gratitud y su impacto en la salud física, siendo los efectos más evidenciados en mejorar la calidad del sueño. Por consiguiente, los hallazgos documentados en la literatura científica reciente sugieren que la gratitud es un elemento significativo para la salud mental.

A pesar de que la gratitud puede ser un concepto secular el cual solamente refleja el agradecimiento de una persona, la literatura científica también establece que dicho constructo puede estar asociado a la $E / R$ (Rosmarin, 2018). La religión puede promover el sentido de gratitud a través del agradecimiento dirigido hacia la divinidad o un ser superior por sus bendiciones y misericordias (Emmons et al., 2008; Moreland, 2019; Rosmarin et al., 2011). Recientemente, Aghababaei et al. (2018) realizaron un multiestudio donde evaluaron las relaciones de gratitud hacia la divinidad y la gratitud disposicional con bienestar y personalidad en una muestra de iraníes musulmanes y cristianos polacos. Los resultados mostraron que la gratitud tiene correlaciones significativas con bienestar psicológico y con factores de personalidad asociados a funcionamiento social y altruismo. Tsang et al. (2011) realizaron un estudio experimental para 
evaluar la relación entre religiosidad intrínseca y gratitud. Los resultados revelaron asociación positiva entre religiosidad intrínseca y gratitud disposicional. Dichos hallazgos sugieren que la $\mathrm{E} / \mathrm{R}$ son dimensiones directamente asociadas a la gratitud, la cual a su vez tiene un impacto positivo en la salud mental. Por lo tanto, se recomienda promover el sentido de gratitud como recurso de afrontamiento a través de las creencias religiosas y espirituales.

\section{Meditación}

La meditación consiste en centrar la atención del individuo en un tema en particular y descentralizarse temporalmente del mundo exterior y los pensamientos relacionados con ello (Lutz et al., 2008). Esta técnica se realiza a través de una variedad de ejercicios de relajación tales como, respiración, repetición de sonidos, observación del pensamiento, entre otros, los cuales buscan promover el bienestar. En la revisión sistemática y metaanálisis de Chen et al. (2012) se identificaron 36 ensayos clínicos aleatorizados del efecto de la meditación en la ansiedad. Los resultados demostraron que las intervenciones basadas en meditación fueron eficaces en la reducción de síntomas de ansiedad. Estos hallazgos son consistentes con el metaanálisis de Goyal et al. (2014) el cual evaluó 47 ensayos clínicos de programas de meditación en pacientes con depresión, ansiedad, dolor crónico, entre otras condiciones. Los resultados sugirieron que los programas basados en meditación podían reducir sintomatología asociada a depresión, ansiedad y dolor crónico. También, existen otras técnicas psicoterapéuticas de base espiritual y religiosa que pueden ser útiles como recursos de afrontamiento para el manejo adaptativo de eventos estresantes tales como, la asistencia a servicios religiosos, participación en diversas actividades junto a una comunidad de fe, memorización de textos sagrados, entre otros (Koenig, 2018; Pearce et al., 2015a; 2016).

\section{CONCLUSIÓN}

A lo largo de este escrito, se ha discutido literatura actualizada y relevante sobre cómo la $\mathrm{E} / \mathrm{R}$ podrían impactar en múltiples formas la salud mental de los individuos. También, se ha resaltado la importancia de los recursos psicoterapéuticos de base espiritual y religiosa como estrategias de afrontamiento ante el COVID-19 y el distanciamiento social, dado que ambos aspectos han causado un desequilibrio en la vida física, social, emocional y espiritual a nivel mundial. Las estrategias psicoterapéuticas de base espiritual y religiosa han mostrado ser beneficiosas cuando se utilizan con fines psicoterapéuticos y no dogmáticos para el manejo de situaciones estresantes, de angustia y crisis. Si bien es cierto que en tiempos de crisis las personas podrían experimentar un debilitamiento en su fe y crisis espirituales/religiosas, también es cierto que la $\mathrm{E} / \mathrm{R}$ han mostrado ser factores protectores al promover apoyo, consuelo, sanación y bienestar en una cantidad significativa de personas con creencias espirituales y religiosas. No hay dudas que una cantidad significativa de personas necesitarán recibir servicios de salud mental durante la pandemia del COVID-19, así como después de que la situación actual se normalice debido a síntomas postraumáticos. Ciertamente puede haber personas pertenecientes a una comunidad de fe que hayan perdido familiares, amigos, estabilidad financiera, emocional y espiritual.

Por consiguiente, es fundamental que los profesionales de la salud mental estén alertas y a la vanguardia de estas necesidades, ya que podrían manifestarse síntomas psicológicos que no estén relacionados directamente a la emergencia del COVID-19, sino a la falta de un lugar seguro que brinde apoyo social como lo son las congregaciones religiosas. Conocer el impacto de la $\mathrm{E} / \mathrm{R}$ en la vida de las personas, sus creencias y cómo se perciben en el área espiritual, podría ayudar al manejo adaptativo de pensamientos, emociones y angustia que estén experimentando. Por esto, es responsabilidad de los profesionales de la salud mental desarrollar competencias en el campo de la psicología de la religión y la espiritualidad para lograr conceptualizar cómo estas dimensiones podrían impactar positiva y negativamente el bienestar de las personas dentro de los tiempos de incertidumbre que actualmente estamos viviendo.

Financiamiento: La presente investigación no fue financiada por alguna entidad ni patrocinador.

Conflicto de Intereses: No existen conflictos de intereses de parte de los autores de la investigación.

Aprobación de la Junta Institucional para la Protección de Seres Humano en la Investigación: No requerida.

Consentimiento o Asentimiento Informado: No aplica.

Proceso de Revisión: Este estudio ha sido revisado por pares externos en modalidad de doble ciego. 


\section{REFERENCIAS}

Abu-Raiya, H., Pargament K.I., Krause N., Ironson, G. (2015). Robust links between religious/spiritual struggles, psychological distress, and well-being in a national sample of American adults. American Journal of Orthopsychiatry, 85(6), 565-575. https://doi.org/10.1037/ort0000084

Aghababaei, N., Błachnio, A., \& Aminikhoo, M. (2018) The relations of gratitude to religiosity, well-being, and personality. Mental Health, Religion $\mathcal{E}$ Culture, 21(4), 408-417. https://doi.org/10.1080/13674676.2018.1504904

American Psychiatric Association. (2013). Diagnostic and statistical manual of mental disorders: DSM-5. American Psychiatric Association.

Anderson, N., Heywood-Everett, S., Siddiqi, N., Wright, J., Meredith, J., \& McMillan, D. (2015). Faith-adapted psychological therapies for depression and anxiety: Systematic review and meta-analysis. Journal of Affective Disorders, 176, 183-196. https://doi.org/10.1016/j.jad.2015.01.019

Ano, G. G., \& Pargament, K. I. (2013). Predictors of spiritual struggles: An exploratory study. Mental Health, Religion \& Culture, 16, 419 - 434. http://dx.doi.org/10.1080/13674676.2012.680434

Ano, G. G., \& Vasconcelles, E. B. (2005). Religious coping and psychological adjustment to stress: A meta-analysis. Journal of Clinical Psychology, 61, 461-480. https://doi.org/10.1002/jclp.20049

Association of Spiritual, Ethical, and Religious Values in Counseling. (2009). Competencies for addressing spiritual and religious issues in counseling. http://www.aservic.org/wpcontent/uploads/2010/04/Spiritual-Competencies-Printerfriendly1.pdf

Aten, J. D., Smith, W. R., Davis, E. B., Van-Tongeren, D. R., Hook, J. N., Davis, D. E.,...\& Hill, P. C. (2019). The psychological study of religion and spirituality in a disaster context: A systematic review. Psychological Trauma: Theory, Research, Practice, and Policy, 11(6), 597-613. https://doi.org/10.1037/tra0000431

Beaglehole, B., Mulder, R.T., Frampton, C.M., Boden, J.M., Newton-Howes, G., \& Bell, C.J. (2018). Psychological distress and psychiatric disorder after natural disasters: Systematic review and meta-analysis. The British Journal of Psychiatry, 213(6), 716722. https://doi.org/10.1192/bjp.2018.210

Best, M., Butow, P., \& Olver, I. (2016). Doctors discussing religion and spirituality: A systematic literature review. Palliative Medicine, 30(4), 327-337. https://doi.org/10.1177/0269216315600912

Boggiss, A. L., Consedine, N. S., Brenton-Peters, J. M., Hofman, P. L., \& Serlachius, A. S. (2020). A systematic review of gratitude interventions: Effects on physical and health behaviors. Journal of Psychosomatic Research, 135. https://doi.org/10.1016/j.jpsychores.2020.110165

Bonelli, R.M. \& Koenig, H.G. (2013). Mental disorders religion and spirituality 1990 to 2010: A systematic evidence-based review. Journal of Religion and Health, 52(2), 657-673. https://doi.org/10.1007/s10943-013-9691-4

Captari, L. E., Hook, J. N., Hoyt, W., Davis, D. E., McElroy-Heltzel, S. E., \& Worthington, E. L., Jr. (2018). Integrating clients' religion and spirituality within psychotherapy: A comprehensive meta-analysis. Journal of Clinical Psychology, 74(11), 1938-1951. https://doi.org/10.1002/jclp.22681

Chen, K., \& Berger, C. (2012). Meditative therapies for reducing anxiety: A systematic review and meta-analysis of randomized controlled trials. Depression and Anxiety, 29(7), 545-562. https://doi.org/10.1002/da.21964

Currier, J. M., \& Eriksson, C. B. (2017). Trauma and spirituality: Empirical advances in an understudied area of community experience. Journal of Prevention $\mathcal{E}$ Intervention in the Community, 45(4), 231-237. https://doi.org/10.1080/10852352.2016.1197755

Daher, N., Koenig, H.G., Pearce, M.J., Nelson, B., Shaw, S.F., Berk, L.S., Belinger, D., Robins, C.J., Cohen, H.J., \& King, M.B. (2016) Effects of Religious vs. Conventional Cognitive-Behavioral Therapy on purpose in life in clients with Major Depression and chronic medical illness: A randomized clinical trial. International. Journal of Existential Psychology \& Psychotherapy, 6(1). https://www.meaning.ca/web/wp-content/uploads/2016/02/172-13-440-1-10-20171211.pdf

de-Oliveira-Maraldi, E. (2020). Response bias in research on religion, spirituality and mental health: A critical review of the literature and methodological recommendations. Journal of Religion and Health, 59(2), 772-783. https://doi.org/10.1007/s10943018-0639-6

Ellison, C.G., Bradshaw, M., Flanelly, K.J., \& Galek, K.C. (2014). Prayer, attachment to God, and symptoms of anxiety related disorder among US adults. Sociology of Religion, 75(2), 208-233.

Emmons, R. A., Barrett, J. L., \& Schnitker, S. A. (2008). Personality and the capacity for religious and spiritual experience. In O. P. John, R. W. Robins, \& L. A. Pervin (Eds.), Handbook of personality: Theory and research (p. 634-653). Guilford.

Emmons, R. A., \& Shelton, C. M. (2002). Gratitude and the science of positive psychology. In C. R. Snyder \& S. J. Lopez (Eds.), Handbook of positive psychology (p. 459-471). Oxford University Press.

Exline, J. J. (2013). Religious and spiritual struggles. En K. I. Pargament, J. J. Exline, \& J. W. Jones (Eds.), APA handbook of psychology, religion, and spirituality (Vol. 1: Context, theory, and research) (p. 459-475). American Psychological Association. https://doi.org/10.1037/14045-025

Fardin, M.A. (2020). COVID-19 Epidemic and spirituality: A review of the benefits of religion in times of crisis. Jundishapur Journal of Chronic Disease Care, 9(2). https://doi.org/10.5812/jjcdc.104260

Gonçalves, J. P. B., Lucchetti, G., Menezes, P. R., \& Vallada, H. (2015). Religious and spiritual interventions in mental health care: A systematic review and meta-analysis of randomized controlled clinical trials. Psychological Medicine, 45(14), 2937-2949. https://doi.org/10.1017/S0033291715001166

González-Rivera, J.A. (2015). Espiritualidad en la clínica: Integrando la espiritualidad en la psicoterapia y la consejería. Ediciones Psicoespiritualidad.

Goyal, M., Singh, S., Sibinga, E. M. S., Gould, N. F., Rowland-Seymour, A., Sharma, R., et al. (2014). Meditation programs for psychological stress and well-being: A systematic review and meta-analysis. JAMA Internal Medicine, 174(3), 357-368. 
https://doi.org/10.1001/jamainternmed.2013.13018

Griffin, G. (2020). Defining trauma and a trauma-informed COVID19 response. Psychological Trauma: Theory, Research, Practice, and Policy, 12(1), S279-S280. https://doi.org/10.1037/tra0000828

Harris, K. A., Randolph, B. E., \& Gordon, T. D. (2016). What do clients want? Assessing spiritual needs in counseling: A literature review. Spirituality in Clinical Practice, 3(4), 250-275. https://doi.org/10.1037/scp0000108

Hathaway, W. L., Scott, S. Y., \& Garver, S. A. (2004). Assessing religious/ spiritual functioning: A neglected domain in clinical practice? Professional Psychology: Research and Practice, 35, 97104. https://doi.org/10.1037/0735-7028.35.1.97

Hook, J. N., Worthington, E. L., Davis, D. E., Jennings, D. J., Gartner, A. L., \& Hook, J. P. (2010). Empirically supported religious and spiritual therapies. Journal of Clinical Psychology, 66(1), 4672. https://doi.org/10.1002/jclp.20626

Horesh, D., \& Brown, A. D. (2020b). Traumatic stress in the age of COVID-19: A call to close critical gaps and adapt to new realities. Psychological Trauma: Theory, Research, Practice, and Policy, 12(4), 331-335. https://doi.org/10.1037/tra0000592

Hossain, M.M., Sultana, A., Purohit, N. (2020a). Mental health outcomes of quarantine and isolation for infection prevention: A systematic umbrella review of the global evidence. Epidemiology and Health, 42. https://doi.org/10.4178/epih.e2020038

Hossain, M. M., Tasnim,S., Sultana, A., Faizah, F., Mazumder, H., Zou, L.,...\& Ma, P. (2020b). Epidemiology of mental health problems in COVID-19: A review. Research, 9, 636. https://doi.org/10.12688/f1000re-search.24457.1

Jans-Beken, L., Jacobs, N., Janssens, M., Peeters, S., Reijnders, J., Lechner, L., \& Lataster, J. (2019). Gratitude and health: An updated review. The Journal of Positive Psychology. Advance online publication. https://doi.org/10.1080/17439760.2019.1651888

Kick, K. A., \& McNitt, M. (2016). Trauma, spirituality, and mindfulness: Finding hope. Social Work \& Christianity, 43(3), 97-108.

Koenig, H.G. (2008). Concerns about measuring "spirituality" in research. The Journal of nervous and mental disease, 196(5), 349-55. https://doi.org/10.1097/nmd.0b013e31816ff796

Koenig, H. G. (2009). Research on religion, spirituality, and mental health: A review. The Canadian Journal of Psychiatry, 54(5), 283291. https://doi.org/10.1177/070674370905400502

Koenig, H.G. (2012). Religion, spirituality, and health: The research and clinical implications. International Scholarly Research Network, 1, 1-33. https://doi.org/10.5402/2012/278730

Koenig H. G. (2015). Religion, spirituality, and health: a review and update. Advances in mind-body Medicine, 29(3), 19-26.

Koenig, H.G. (2018). Religion and mental health: Research and clinical applications. Academic Press.

Koenig, H.G. (2020). Maintaining health and well-being by putting faith into action during the COVID-19 pandemic. Journal of Religion Health, 59(5), 2205-2214. https://doi.org/10.1007/s10943-020-01035-2

Koenig, H. G., Al-Zaben, F., \& VanderWeele, T. J. (2020). Religion and psychiatry: Recent developments in research. British Journal of Psychiatry Advances, 26(5), 262-272. https://doi.org/10.1192/bja.2019.81

Koenig, H. G., Pearce, M. J., Nelson, B., \& Daher, N. (2015). Effects of religious versus standard cognitive-behavioral therapy on optimism in persons with major depression and chronic medical illness. Depression and Anxiety, 32(11), 835-842. https://doi.org/10.1002/da.22398

Koenig, H. G., \& Pritchett, J. (1998). Religion and psychotherapy. In H. G. Koenig (Ed.), Handbook of religion and mental health ( $\mathrm{p}$. 323-336). Academic Press. https://doi.org/10.1016/B978012417645-4/50089-4

Lim, C., Sim K., Renjan, V., Sam, H. F., \& Quah, S.L. (2014). Adapted cognitive-behavioral therapy for religious individuals with mental disorder: A systematic review. Asian Journal of Psychiatry, 9, 3-12. https://doi.org/10.1016/j.ajp.2013.12.011

Lorenzo-Ruiz, A., Díaz, K., \& Zaldívar, D. (2020). La psicología como ciencia y profesión en el afrontamiento del COVID-19. Revista Caribeña de Psicología, 4(2), 153-165. https://doi.org/10.37226/rcp.v4i2.4815

Lun, V. M.-C., \& Bond, M. H. (2013). Examining the relation of religion and spirituality to subjective well-being across national cultures. Psychology of Religion and Spirituality, 5(4), 304-315. https://doi.org/10.1037/a0033641

Lutz, A., Slagter, H. A., Dunne, J. D., \& Davidson, R. J. (2008). Attention regulation and monitoring in meditation. Trends in $\mathrm{Cog}-$ nitive Sciences, $12(4), \quad 163-169$ https://dx.doi.org/10.1016\%2Fj.tics.2008.01.005

Moreland, J.P. (2019). Finding Quiet: My story of overcoming anxiety and the practices that brought peace. Zondervan

Oh, P. J., \& Kim, Y. H. (2012). Meta-analysis of spiritual intervention studies on biological, psychological, and spiritual outcomes. Journal of Korean Academy Nursing, 42(6), 833-842. https://doi.org/10.4040/jkan.2012.42.6.833

Oman D., Syme S.L. (2018). Weighing the evidence: What is revealed by $100+$ meta-analyses and systematic reviews of religion/spirituality and health? In D. Oman (Ed.) Why religion and spirituality matter for public health. religion, spirituality and health: A social scientific approach, (vol 2. p.261-281) Springer. https://doi.org/10.1007/978-3319-73966-3_15

Oxhandler, H., Ellor, J., \& Stanford, M. (2018). Client attitudes toward integrating religion and spirituality in mental health treatment: Scale development and client responses. Social Work, 63(4), 337-346. https://doi.org/10.1093/sw/swy041

Pagán-Torres, O.M., Reyes-Estrada, M. \& Cumba-Avilés, E. (2017). Religión, espiritualidad y Terapia Cognitivo Conductual: Una reseña actualizada. Revista Salud y Conducta Humana, 4(1), 1334. http://rsych.com/blog/

Paloutzian, R.F., \& Park, C.L. (2013). Handbook of the Psychology of Religion and Spirituality. The Guilford Press.

Pargament, K. I., Mahoney, A., \& Shafranske, E. P. (2013). APA handbook of psychology, religion, and spirituality (Vol. 2): An applied psychology of religion and spirituality. American Psychological Association. https://doi.org/10.1037/14046-000 
Pargament, K. I., Smith, B. W., Koenig, H. G., \& Perez, L. (1998). Patterns of positive and negative religious coping with major life stressors. Journal for the Scientific Study of Religion, 37(4), 710-724. https://doi.org/10.2307/1388152

Park, C. L., Currier, J. M., Harris, J. I., \& Slattery, J. M. (2017). Reciprocal relationships between spirituality and trauma. In Trauma, meaning, and spirituality: Translating research into clinical practice. (p. 39-54). American Psychological Association. https://doi.org/10.1037/15961-003

Park, C.L., Masters, K.S., Salsman, J.M., Wachholtz, A., Clements, A.D., Salmoirago-Blotcher, E...Wischenka, D.M. (2017). Advancing our understanding of religion and spirituality in the context of behavioral medicine. Journal of Behavioral Medicine, 40, 39-51. https://doi.org/10.1007/s10865-016-9755-5

Paukert, A., Phillips, L., Cully, J., Romero, C., \& Stanley, M. (2011). Systematic review of the effects of Religion-Accommodative Psychotherapy for depression and anxiety. Journal of Contemporary Psychotherapy, 41, 99-108. https://doi.org/10.1007/s10879010-9154-0

Pearce, M. (2016). Cognitive behavioral therapy for christians with depression: A practical tool-based primer. Templeton Foundation Press.

Pearce, M., Koenig, H. G., Robins, C. J., Bruce, N., Shaw, S. F., Cohen, H. V., \& King, M. B. (2015a). Religiously integrated cognitive behavioral therapy: A new method of treatment for major depression in patients with chronic medical illness. Psychotherapy, 52(1), 56-66. http://dx.doi.org/10.1037/a0036448

Pearce, M. J., Koenig, H. G., Robins, C. J., Daher, N., Shaw, S. F., Nelson, B., Berk, L. S., Bellinger, D., Cohen, H. J., \& King, M. B. (2015b). Effects of religious versus conventional cognitive-behavioral therapy on generosity in major depression and chronic medical illness: A randomized clinical trial. Spirituality in Clinical Practice, 2(3), 202-215. https://doi.org/10.1037/scp0000076

Peres, J. F. P., Moreira-Almeida, A., Nasello, A. G., \& Koenig, H. G. (2007). Spirituality and resilience in trauma victims. Journal of Religion and Health, 46(3), 343-350. https://doi.org/10.1007/s10943-006-9103-0

Pirutinsky, S., Cherniak, A.D. \& Rosmarin, D.H. (2020). COVID-19, mental health, and religious coping among American orthodox Jews. Journal of Religion and Health, 59, 2288-2301. https://doi.org/10.1007/s10943-020-01070-z

Post, B. C., \& Wade, N. G. (2009). Religion and spirituality in psychotherapy: A practice-friendly review of research. Journal of Clinical Psychology, 65, 131-146. https://doi.org/10.1002/jclp.20563

Prado, T., Caldeira, S. \& Campos de Carvalho, E. (2016). The effect of prayer on patients' health: Systematic literature review. Religions, 7(11), 1-11. https://doi.org/10.3390/rel7010011

Quadri, S.A. (2020). COVID-19 and religious congregations: Implications for spread of novel pathogens. International Journal of Infectious Diseases, 96, 219-221. https://doi.org/10.1016/j.ijid.2020.05.007

Ramos, K., Erkanli, A., \& Koenig, H. G. (2018). Effects of religious versus conventional cognitive-behavioral therapy (CBT) on suicidal thoughts in major depression and chronic medical illness. Psychology of Religion and Spirituality, 10(1), 79-87. https://doi.org/10.1037/rel0000113

Richards, P.S. \& Bergin, A. (2005). A spiritual strategy for counseling and psychotherapy. American Psychological Association.

Richards, P. S., \& Bergin, A. E. (2014). Handbook of psychotherapy and religious diversity (2nd ed.). American Psychological Association.

Rodríguez-Diaz, C.E., Guilamo-Ramos, V., Mena, L., Hall, E., Honermann, B., Crowley, J.S., ...\& Millett G.A. (2020). Risk for COVID19 infection and death among Latinos in the United States: Examining heterogeneity in transmission dynamics. Annals of Epidemiology, 52, 46-53. https://doi.org/10.1016/j.annepidem.2020.07.007

Rosmarin, D.H. (2018). Spirituality, Religion and Cognitive Behavior Therapy. Guilford Press.

Rosmarin, D.H., Pargament, K.I., Pirutinsky, S., Mahoney, A. (2010). A randomized controlled evaluation of a spiritually integrated treatment for subclinical anxiety in the Jewish community delivered via the Internet. Journal of Anxiety Disorders, 24(7), 799-808. https://doi.org/10.1016/j.janxdis.2010.05.014

Rosmarin, D. H., Pirutinsky, S., Cohen, A. B., Galler, Y., \& Krumrei, E. J. (2011). Grateful to God or just plain grateful? A comparison of religious and general gratitude. The Journal of Positive $\begin{array}{lll}\text { Psychology, 6(5), 389-396. } & \text { 60 }\end{array}$ https://doi.org/10.1080/17439760.2011.596557

Safara, M. \& Bhatia, M.S. (2009). Prayer Therapy. Delhi Psychiatry Journal, 12(1), 67-73.

Saunders, S. M., Miller, M. L., \& Bright, M. M. (2010). Spiritually conscious psychological care. Professional Psychology: Research and Practice, 41, 355-362. https://doi.org/10.1037/a0020953

Sedlar, A.E.; Stauner, N.; Pargament, K.I.; Exline, J.J.; Grubbs, J.B.; Bradley, D.F. (2018). Spiritual struggles among atheists: links to psychological distress and well-being. Religions, 9(8). 242. https://doi.org/10.3390/rel9080242

Smith, T. B., Bartz, J., \& Richards, P. S. (2007). Outcomes of religious and spiritual adaptations to psychotherapy: A meta-analytic review. Psychotherapy Research, 17(6), 643-655. http://www.tandfonline.com/doi/abstract/10.1080/10503300701250347

Stanley, M. A., Bush, A. L., Camp, M. E., Jameson, J. P., Phillips, L. L., Barber, C. R., Zeno, D., Lomax, J. W., \& Cully, J. A. (2011). Older adults' preferences for religion/spirituality in treatment for anxiety and depression. Aging \& mental health, 15(3), 334343. https://doi.org/10.1080/13607863.2010.519326

Starnino, V. R. (2016). When trauma, spirituality, and mental illness intersect: A qualitative case study. Psychological Trauma: Theory, Research, Practice, and Policy, 8(3), 375-383. https://doi.org/10.1037/tra0000105

Stephens, N. M., Fryberg, S. A., Markus, H. R., \& Hamedani, M. G. (2013). Who explains Hurricane Katrina and the Chilean earthquake as an act of God? The experience of extreme hardship predicts religious meaning-making. Journal of Cross-Cultural $\begin{array}{lll}\text { Psychology, } & \text { 64(4), }\end{array}$ 
https://doi.org/10.1177/0022022112454330

Sulkowski, L., \& Ignatowski, G. (2020). Impact of COVID-19 pandemic on organization of religious behaviour in different christian denominations in Poland. Religions. 11(5), 254. https://doi.org/10.3390/rel11050254

Spika, B., \& Ladd, K.L. (2012). The psychology of prayer: A scientific approach. Guilford Press.

Tan, S.Y. (1996). Religion in clinical practice: Implicit and explicit integration. In E. P. Shafranske (Ed.), Religion and the clinical practice of psychology (p. 365-387). American Psychological Association. https://doi.org/10.1037/10199-013

Tan, S.Y., \& Johnson, W. B. (2005). Spiritually oriented cognitive behavioral therapy. In L. Sperry, \& E. P. Shafranske (Eds.), Spiritually oriented psychotherapy (p. 77-103). American Psychological Association. https://doi.org/10.1037/10886-004

Tsang, J.-A., Schulwitz, A., \& Carlisle, R. D. (2012). An experimental test of the relationship between religion and gratitude. Psychology of Religion and Spirituality, 4(1), 40-55. https://doi.org/10.1037/a0025632

Substance Abuse and Mental Health Services Administration (2014). SAMHSA's Concept of Trauma and Guidance for a TraumaInformed Approach. https://ncsacw.samhsa.gov/userfiles/files/SAMHSA_Trauma.pdf

Van-Hook, M. P. (2016). Spirituality as a potential resource for coping with trauma. Social Work \& Christianity, 43(1), 7-25.

Van-Tongeren, D. R., Sanders, M., Edwards, M., Davis, E. B., Aten, J. D., Ranter, J. M., Tsarouhis, A., Short, A., Cuthbert, A., Hook,
J. N., \& Davis, D. E. (2019). Religious and spiritual struggles alter God representations. Psychology of Religion and Spirituality, 11(3), 225-232. https://doi.org/10.1037/rel0000173

Vieten, C., Scammell, S., Pilato, R., Ammondson, I., Pargament, K. I., \& Lukoff, D. (2013). Spiritual and religious competencies for psychologists. Psychology of Religion and Spirituality, 5(3), 129144. https://doi.org/10.1037/a0032699

Wade, N. G., Worthington, E. L., Jr., \& Vogel, D. L. (2007). Effectiveness of religiously tailored interventions in Christian therapy. Psychotherapy Research, 17(1), 91-105. https://doi.org/10.1080/10503300500497388

World Health Organization. (2020). Practical considerations and recommendations for religious leaders and faith-based communities in the context of COVID-19. https://www.who.int/publications/i/item/practical-considerations-and-recommendations-for-religious-leaders-and-faith-based-communities-in-the-context-of-covid19?gclid=Cj0KCQjwpZT5BRCdARIsAGEX0zka3FMLQF3HReOOiJIcocuMgO9JRw0CsGmcZagZgP-ZGCiQ2q8Sd7UaAgMUEALw_wcB

Worthington, E. L., Jr., Hook, J. N., Davis, D. E., \& McDaniel, M. A. (2011). Religion and spirituality. In J. C. Norcross (Ed.), Psychotherapy relationships that work: Evidence-based responsiveness ( $\mathrm{p}$. 402-419). Oxford University Press. https://doi.org/10.1093/acprof:oso/9780199737208.003.0020

Yamada, A.-M., Lukoff, D., Lim, C. S. F., \& Mancuso, L. L. (2020). Integrating spirituality and mental health: Perspectives of adults receiving public mental health services in California. Psychology of Religion and Spirituality, 12(3), 276-287. https://doi.org/10.1037/rel0000260 